Document downloaded from:

http://hdl.handle.net/10251/65558

This paper must be cited as:

García Baldoví, H.; Valencia Valencia, S.; Alvaro Rodríguez, MM.; Abdullah, AM.; García Gómez, H. (2015). Highly fluorescent C-dots obtained by pyrolysis of quaternary ammonium ions trapped in all-silica ITQ-29 zeolite. Nanoscale. 7(5):1744-1752.

doi:10.1039/C4NR05295A.

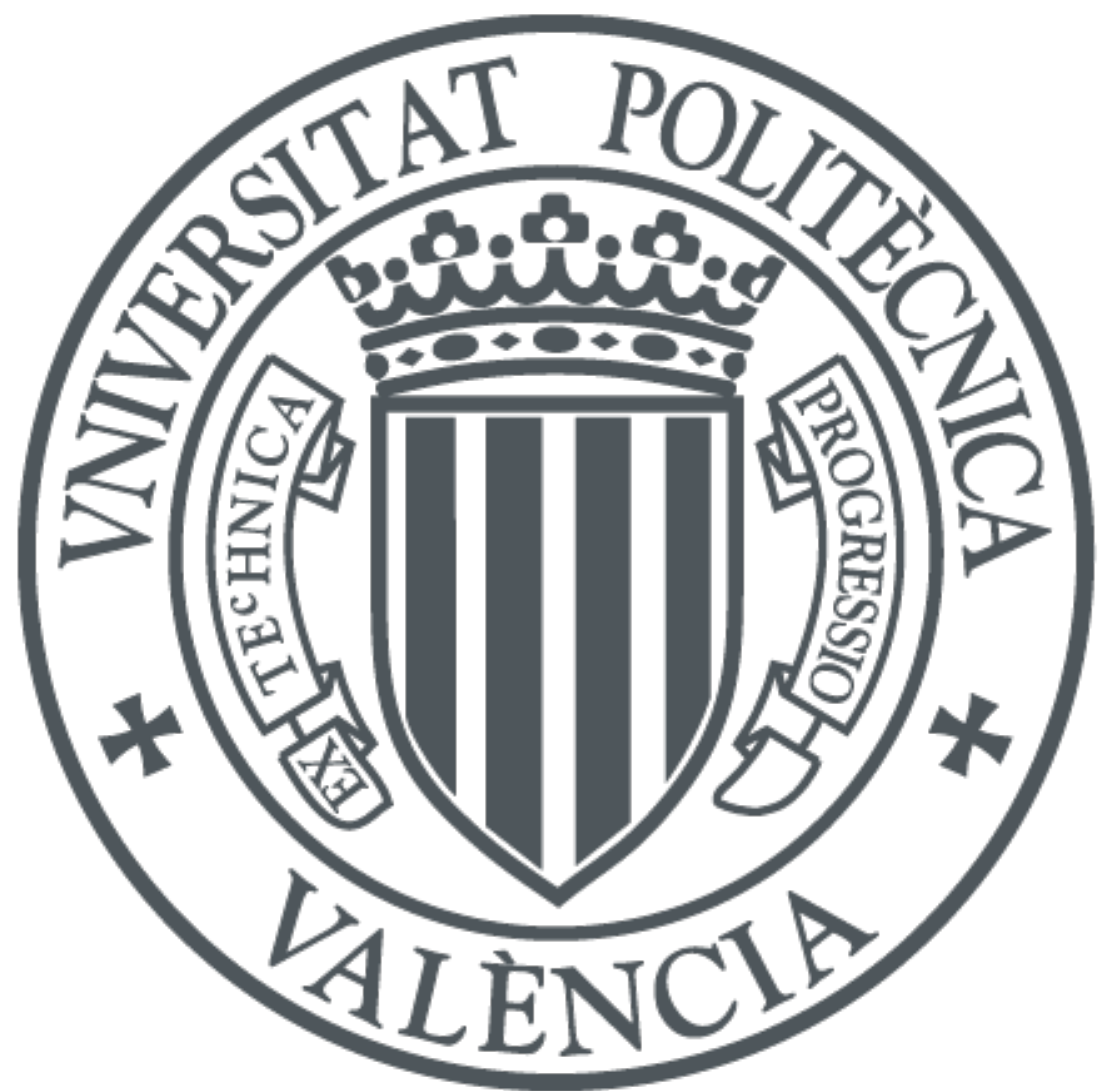

The final publication is available at

http://dx.doi.org/10.1039/c4nr05295a

Copyright Royal Society of Chemistry

Additional Information 


\title{
Highly fluorescent C-dots obtained by pyrolysis of quaternary ammonium ions trapped in all-silica ITQ-29 zeolite.
}

\author{
Herme G Baldovi, ${ }^{1}$ Susana Valencia, ${ }^{1}$ Mercedes Alvaro, ${ }^{1}$ Abdullah M. Asiri ${ }^{2}$ and Hermenegildo Garcia ${ }^{1,2, *}$
}

\begin{abstract}
.
C-dots obtained in homogeneous phase may exhibit a broad particle size distribution. Formation of C-dots within nanometric reaction cavities could be a methodology to gain control on their size distribution. Among the various possibilities, in the present work, the cavities of small pore size zeolites have been used to confine C-dots generated by pyrolysis of the organic structure directing agent present in the synthesis of these crystalline aluminosilicates. To explore this methodology, ITQ-29 zeolite having a Linde type A (LTA) structure was prepared as pure silica with 4-methyl-2,3,6,7-tetrahydro-1H,5H-pyrido[3.2.1-ij]quinolinium as organic structure directing agent. Pyrolysis under inert atmosphere at $550{ }^{\circ} \mathrm{C}$ of a pure-silica ITQ-29 sample (cubic particles of $4 \mu \mathrm{m}$ edge) renders a highly fluorescent zeolite containing about $15 \mathrm{wt} \%$ of carbonised residue. While other small pore zeolite, ITQ-12 (ITW), also renders photoluminescence C-dots under similar conditions, medium or large pore zeolites, such as silicalite (MFI) or pure silica Beta (BEA), failed to produce fluorescent powders under analogous thermal treatment and only decomposition and complete removal of the corresponding quaternary ammonium ion templates was observed for these zeolites. Dissolution of the pyrolysed ITQ-29 zeolite framework and extraction of the carbon residue with ethyl acetate has allowed characterisation of C-dots with particle size between 5-12 nm and a photoluminescence quantum yield of 0.4 upon excitation at $350 \mathrm{~nm}$ that is among the highest reported for non surface functionalized C-dots. The photoluminescence varies with the excitation wavelength and is quenched by oxygen. Pyrolysed ITQ-29 powders can act as fluorescent oxygen sensor.
\end{abstract}

Keywords: Carbon Dots, Photoluminescent Quantum Dots, Zeolites as templates, Oxygen Sensor, ITQ-29 as C-dot Precursor

\section{Introduction.}

Fluorescent nanoparticles (NPs) have a wide range of applications that span from photochemical probes, chemical sensors, photocatalysis, imaging and bioimaging, and electroluminescence among others. ${ }^{-7}$ Due to the large potential in the possibilities to control their synthesis as well as the availability of their precursors, fluorescent carbon quantum dots (C-dots) have become a field of much current interest. ${ }^{8}$ Fluorescent $\mathrm{C}$-dots could be a viable alternative to conventional quantum dots based on sulphides or chalcogenides of transition metals that in the most widely employed cases such CdSe, CdTe and $\mathrm{PbS}$ are highly toxic. ${ }^{9-15}$

Two general methodologies, either top down ${ }^{11,16}$ or bottom up ${ }^{17,18}$ have been applied for the synthesis of C-dots. Among these reported synthetic methodologies for the preparation of fluorescent $\mathrm{C}$-dots those that have attracted more attention due to their simplicity have been hydrothermal carbonisation under various conditions of suitable molecular or polymeric precursors, ${ }^{19-21}$ but also laser ablation ${ }^{16,}{ }^{22}$ and arc discharge $^{23}$ have been widely employed. One of the main problems in the synthesis of C-dots is the formation of particles with uniform properties and narrow particle size distribution.

Generally hydrothermal carbonisation of organic compounds is carried out in homogeneous liquid phase and control of the dimensions of the C-dots is only possible by optimisation of the experimental conditions. In the present work we have taken advantage of the confined space of a rigid zeolitic LTA framework to prepare highly fluorescent C-dots. Since most frequently zeolites are prepared by employing structure directing agents that template the polymerisation of silica leading to the creation of channels and cavities, ${ }^{24-26}$ we reasoned that after the synthesis the entrapped organic molecules can be suitable precursors for the preparation of C-dots of uniform dimension distribution. Zeolites are highly crystalline solids and the location and position of the organic templates inside the microporous system of the inorganic framework is strictly defined. Therefore, the solid can be viewed as a rigid matrix confining the carbonisation of the template in an initially defined selfassembled arrangement and providing site isolation of individual carbonised quantum dots. In addition, the process can be carried out in the solid state in the absence of any solvent. The influence on the photoluminescence properties of C-dots due to possible surface modification by the solvent molecules would be minimized in this way. The inertness and robustness of the zeolitic structure in thermal treatments should make them interfere less in the formation of entrapped C-dots. There are precedents in the literature in which crystalline aluminophosphate AlPO-5 with monodirectional structure constituted by channels containing tripropylammonium ion has been used for the preparation of C-dots, ${ }^{27}$ but this zeotype AlPO5 has been found better suited for the formation of single wall carbon nanotubes with very narrow diameter. ${ }^{28-35}$

\section{Results and Discussion.}

For the present study, four different zeolites all of them being synthesised in the absence of $\mathrm{Al}$ as pure silica materials 
using structure directing agent and having large (zeolite Beta), medium (Silicalite) and small (ITQ-29 or ITQ-12) pore size were selected. Table 1 summarises the main structural data of the four zeolites, together with the chemical structure of the corresponding quaternary ammonium cation employed in their synthesis. The four zeolites studied were prepared in their pure silica forms in order to avoid the stronger interaction of the organic structure directing agents with the charged aluminosilicate frameworks.

Table 1. Geometry, pore size and structure of the quaternary ammonium cation of the pure silica zeolites used in the preparation of $\mathrm{C}$-dots.

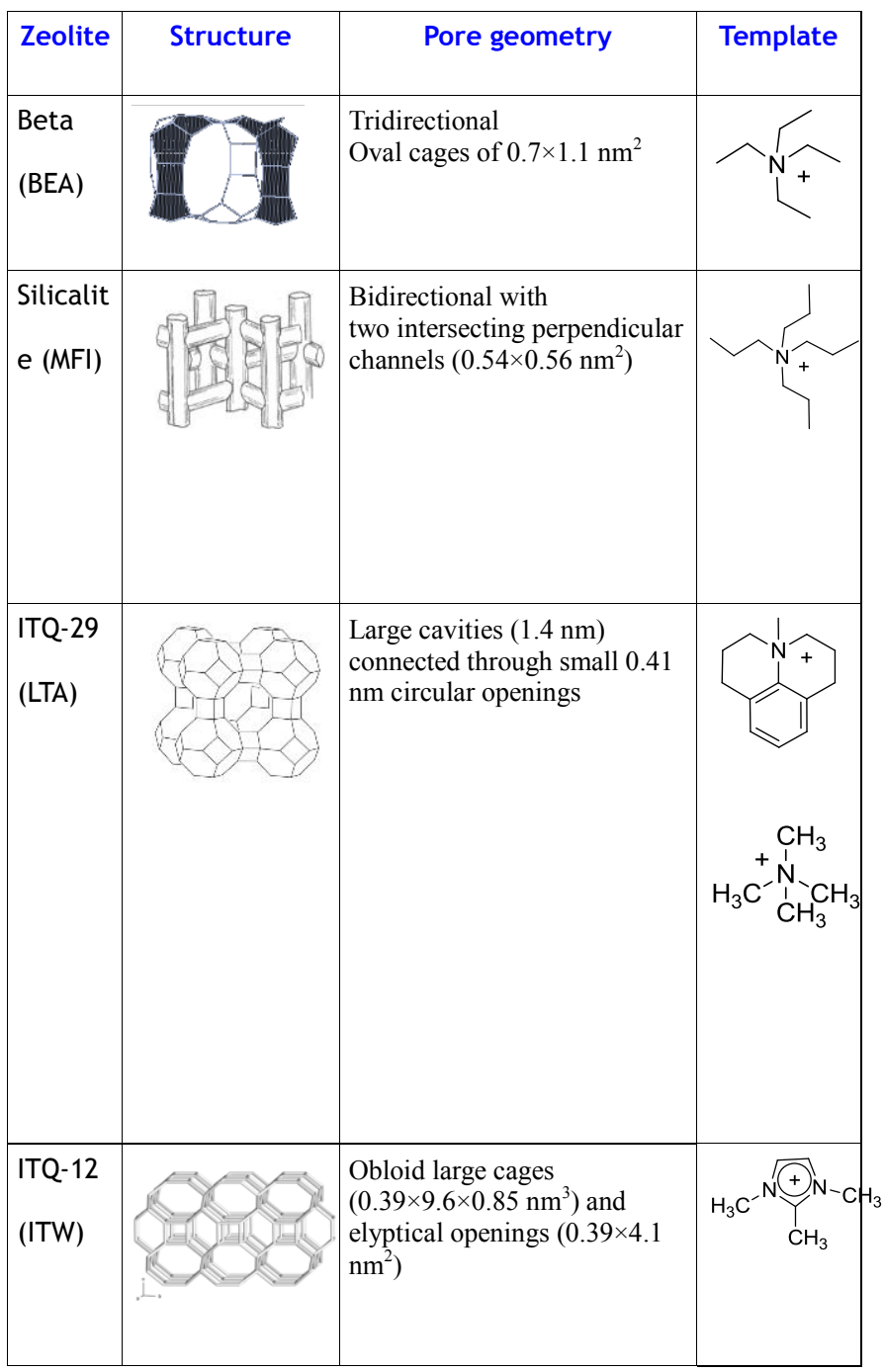

All the attempts to form C-dots using large or medium pore sized zeolites by thermal treatment under inert atmosphere met with failure. Upon increasing the temperature of the thermal treatment of these two types of zeolites in the range 350 to 700 ${ }^{\circ} \mathrm{C}$, decomposition and complete removal of the template was observed as it was deduced from the absence of carbon element in the resulting pyrolysed solids. This failure could be due to a combination of effects including dimensions and geometry of the pores and the structure of the quaternary ammonium ion used as template.
In contrast, in the case of small pore size ITQ-29 having a LTA-structure type with large cavities and small pore openings of $0.41 \mathrm{~nm}$, we were able to obtain a material whose intense photoluminescence indicates the formation of C-dots. Thus, an all-silica ITQ-29 sample containing 4-methyl-2,3,6,7tetrahydro-1H,5H-pyrido[3.2.1-ij]quinolinium and tetramethylammonium $\left(\mathrm{TMA}^{+}\right)$(see chemical structure in Table 1) as structure directing agents was submitted under nitrogen atmosphere to thermal treatment at three different temperatures, namely 350,550 and $750{ }^{\circ} \mathrm{C}$ for different periods of time (Table 2 ). It was found that $24 \mathrm{~h}$ was a suitable time for the pyrolysis under our conditions to achieve samples with a high intensity emission.

Table 2. Influence of the treatment time on the photoluminescence intensity of the resulting pyrolysed ITQ-29.

\begin{tabular}{|c|c|c|c|}
\hline \multirow{2}{*}{ Time (h) } & \multicolumn{3}{|c|}{ Intensity emission (counts) } \\
\cline { 2 - 4 } & $\mathbf{3 5 0}^{\mathbf{}} \mathbf{C}$ & $\mathbf{5 5 0}^{\circ} \mathbf{C}$ & $\mathbf{7 5 0}^{\circ} \mathbf{C}$ \\
\hline 3 & -- & -- & -- \\
\hline $\mathbf{1 2}$ & -- & $<500$ & 3586 \\
\hline $\mathbf{2 4}$ & $<500$ & $\mathbf{9 7 7 8}$ & $\mathbf{5 8 6 8}$ \\
\hline $\mathbf{7 2}$ & $\mathbf{6 1 9 4}$ & 3912 & $<500$ \\
\hline
\end{tabular}

The carbon content of the ITQ-29 samples pyrolysed for $24 \mathrm{~h}$ was determined by combustion chemical analysis. Table 3 shows the $\mathrm{C}, \mathrm{N}$ and $\mathrm{H}$ content of the as-synthesised ITQ-29 zeolite and after been submitted to thermal treatment at the indicated temperature under inert atmosphere. Sample 2 (see Tables 2 and 3) was the solid that exhibited the most intense photoluminescence indicating that there should be an optimal thermal treatment to obtain the strongest emitting C-dots. This enhanced emission could be due to optimal balance among a high $\mathrm{C}$ content of the solid after the pyrolysis and adequate composition of the $\mathrm{C}$-dots in terms of low $\mathrm{H}$ content, while still having a significant $\mathrm{N}$ percentage and high density of fluorophore units generated during the pyrolysis.

Table 3. Analytical data of the as-synthesised and pyrolised ITQ29.

\begin{tabular}{|c|c|c|c|c|}
\hline ITQ-29 & $\begin{array}{c}\text { Pyrolysis } \\
\text { temperature } \\
\left({ }^{\circ} \mathrm{C}\right)\end{array}$ & \multicolumn{3}{|c|}{ Percentage (\%) } \\
\hline $\mathbf{0}$ & - & 18.17 & 2.09 & 2.61 \\
$\begin{array}{c}\text { (as- } \\
\text { synthesised) }\end{array}$ & $\mathbf{3}$ & $\mathbf{H}$ & \\
\hline $\mathbf{1}$ & $\mathbf{3 5 0}$ & 16.64 & 1.98 & 2.18 \\
\hline $\mathbf{2}$ & $\mathbf{5 5 0}$ & 14.64 & 1.59 & 0.84 \\
\hline $\mathbf{3}$ & $\mathbf{7 5 0}$ & 0.39 & 0.08 & 0.05 \\
\hline
\end{tabular}

As it can be seen in Table 3, the general trend is that the organic content decreases upon increasing the pyrolysis 
temperature. Pyrolysis at $750{ }^{\circ} \mathrm{C}$ results in the almost complete removal of the organic material.

Further tuning of the pyrolysis temperature was made in the range from 450 to $600{ }^{\circ} \mathrm{C}$ and the results are given in Table 4. As it can be seen there, while all the pyrolysed samples were highly photoluminescent, the optimal temperature appears to be around $550{ }^{\circ} \mathrm{C}$. The decrease in the content of organic matter (from 550 to $600{ }^{\circ} \mathrm{C}$ ) and the variation in the composition and structure of the $\mathrm{C}$-dots should be responsible for these variations in the emission intensity.

Table 4. Relative emission intensity (excitation wavelength $350 \mathrm{~nm}$ ) for ITQ-29 samples submitted to pyrolysis under argon for $24 \mathrm{~h}$ at the indicated temperature.

\begin{tabular}{|c|c|ccc|}
\hline $\begin{array}{c}\text { Temperature } \\
\left({ }^{\circ} \mathbf{C}\right)\end{array}$ & $\begin{array}{c}\text { Emission } \\
\text { Intensity } \\
\text { (counts) }\end{array}$ & \multicolumn{3}{|c|}{ Percentage (\%) } \\
\hline $\mathbf{4 5 0}$ & 4291 & 16.02 & 1.79 & 1.31 \\
\hline $\mathbf{5 0 0}$ & 6118 & 15.34 & 1.70 & 1.15 \\
\hline $\mathbf{5 5 0}$ & $\mathbf{9 7 7 8}$ & $\mathbf{1 4 . 6 4}$ & $\mathbf{1 . 5 9}$ & $\mathbf{0 . 8 4}$ \\
\hline $\mathbf{6 0 0}$ & 8075 & 12.78 & 1.46 & 0.65 \\
\hline
\end{tabular}

It should be commented that a similar removal of the organic material was observed for the medium and large pore zeolites even at temperatures considerably lower, making unsuccessful the generation of C-dots. We suggest that the success of the carbonisation in ITQ-29 derives from the combination of a pore geometry consisting in large cavities with small windows that do not allow diffusion of the structure directing agent (see Table 1) and a conformationally rigid quaternary quinolinium ammonium ion that cannot easily escape out of the cavities without degradation. This suggestion was further supported by preliminary studies submitting another small pore zeolite, namely ITQ-12 with ITW framework (see Table 1), to pyrolysis at $550{ }^{\circ} \mathrm{C}$ for $24 \mathrm{~h}$ under the same conditions as ITQ29. While pristine ITQ-12 does not emit upon excitation at 350 $\mathrm{nm}$, observation of an intense blue emission (maxima ca. $430 \mathrm{~nm}$ upon $350 \mathrm{~nm}$ excitation) for pyrolysed ITQ-12 indicates that photoluminescent species have been formed also in this process as in the case of ITQ-29 under study. The amount of C-dots quantified by the percentage of $\mathrm{C}$ in the solid powder was $9.52 \%$ and the emission intensity of pyrolysed ITQ-12 powders is similar as that of pyrolysed ITQ-29 powders measured under the same conditions.

Thermogravimetric (TG) analysis of the pyrolysed samples 1-3 in Table 2 was compared to that of the fresh material 0 (Figure 1). TG profiles were in good agreement with the chemical analysis data showing that the sample pyrolysed at $750{ }^{\circ} \mathrm{C}$ does not almost contain any organic material (curve 3 in Figure 1). In the case of the as-synthesised sample $\mathbf{0}$ or material 1 pyrolysed at $350{ }^{\circ} \mathrm{C}$, differential scanning calorimetry show several decomposition processes. In contrast, for sample $\mathbf{2}$ (plot 2 in Figure 1) calorimetry shows a single well defined combustion process peaking at $630{ }^{\circ} \mathrm{C}$ (see plot 2' in Figure 1), indicating that the residual carbonised organic matter presents a uniform property with respect to aerobic decomposition.

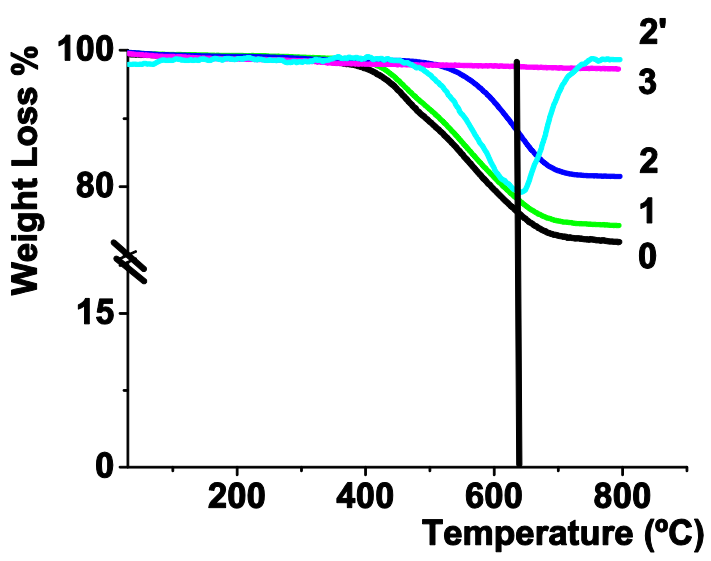

Figure 1. TG profiles under air of samples $\mathbf{0 - 3}$. The differential scanning calorimetry profile of sample $\mathbf{2}$ is also presented (DSC 2 ') to show the uniform nature of the carbonised residue present in ITQ-29 after pyrolysis at $550{ }^{\circ} \mathrm{C}$. The vertical line corresponds to the temperature of $630{ }^{\circ} \mathrm{C}$.

Evidence that pyrolysis at $550{ }^{\circ} \mathrm{C}$ leads to the formation of C-dots trapped in the zeolite matrix was obtained by optical microscopy and emission spectroscopy. Optical microscopy in the fluorescence mode shows that zeolite particles submitted to pyrolysis at $550{ }^{\circ} \mathrm{C}$ exhibit intense yellow fluorescence under blue light illumination (Figure 2, bottom left panel). Sample 2 even fluoresces with red light that is the most appropriate for imaging of cells in biology. This fluorescence is in sharp contrast with the behaviour of the other zeolite samples 0,1 and 3 and indicates that the quinolinium template has been carbonised under these conditions.

Photoluminescence spectra were also recorded upon excitation of sample $\mathbf{2}$ at different wavelengths in the range from 300 to $500 \mathrm{~nm}$. Figure 3 shows the absorption and emission spectra of sample 2 . The variation of the emission spectra as a function of the excitation wavelength is one of the main traits of the C-dots. ${ }^{8}$ We noted that in our case the C-dots should contain some percentage of nitrogen as determined by chemical analysis and that there are precedents indicating that $\mathrm{N}$-doping can enhance the emission intensity of C-dots. ${ }^{36}$ 

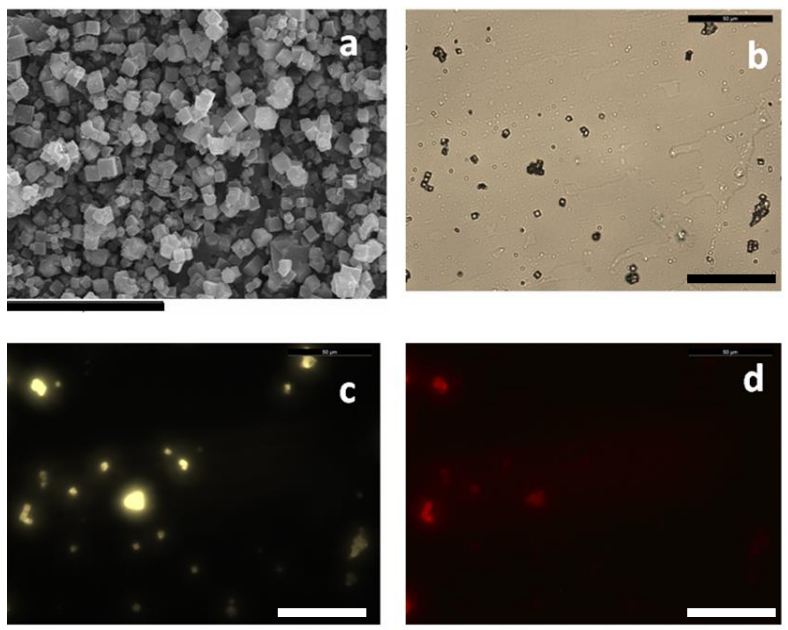

Figure 2. a: Scanning electron microscopy of the ITQ29 batch used in the present study. The scale bar corresponds to $50 \mu \mathrm{m}$; b: Image by optical microscopy of sample 2; c: image of the same sample as in panel top right with blue light $(\lambda<400 \mathrm{~nm})$ illumination recording the image from $450 \mathrm{~nm}$; d: image of the same sample as in panel top right with green light illumination $(\lambda<550 \mathrm{~nm})$ taken the image with light of wavelength longer than $600 \mathrm{~nm}$. Note that the yellow and red colors observed in panels $\mathrm{c}$ and $\mathrm{d}$ are due to emitted light and does not correspond to the visual appearance of the zeolite under white light illumination that is brown. Light source used in the optical microscopy comes from a mercury halide bulb.

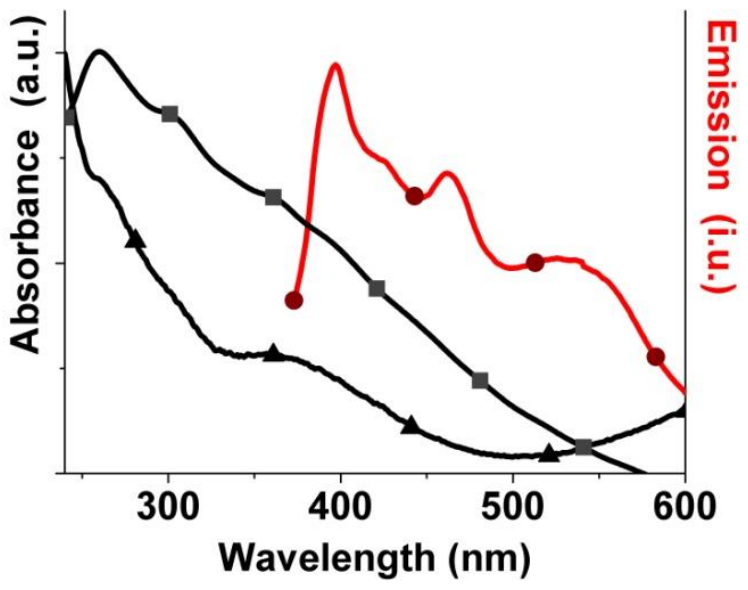

Figure 3. Absorbance UV-Vis spectra $\left(0.7 \mathrm{mg} \mathrm{ml}^{-1}\right.$ of zeolite ITQ-29 treated, in acetonitrile) of samples $\mathbf{0}(\mathbf{\Delta})$ and 2 (๘) and the emission spectrum recorded for sample $\mathbf{2}(\bullet, 0.7 \mathrm{mg}$ $\mathrm{ml}^{-1}$ in acetonitrile) upon excitation at $300 \mathrm{~nm}$.

In order to confirm firmly the formation of C-dots, sample 2 was submitted to dissolution of the silicate framework using concentrated HF. We note that precedents in the literature working with highly reactive polyacetylene and other conducting polymers have shown that HF treatment allows dissolving the zeolite structure without producing changes in the highly reactive conducting organic polymer. ${ }^{37,}{ }^{38}$ In general organic compounds are more stable to HF treatment than zeolite aluminosilicate framework, and in particularly all-silica ITQ-29 that is a relatively unstable material easy to be attacked by $\mathrm{HF}^{39}$ However, caution should be taken considering that HF digestion even at near ambient temperature can produce alteration on the original carbonised residue with respect to the morphology and photoluminescence properties of the entrapped C-dots. To address these possible changes, the influence of the $\mathrm{pH}$ value of the surrounding liquid phase on the emission spectra of sample 2 was studied, observing relatively minor changes on the wavelength emission maximum, the most salient one being the intensity of a medium peak at $450 \mathrm{~nm}$ that almost disappears at acid $\mathrm{pH}$ values.

After dissolution of the zeolite framework, the resulting liquor was extracted with ethyl acetate in order to recover the carbonised residue derived of the template. The organic content with ethyl acetate was strongly photoluminescent indicating that this procedure is adequate to recover $\mathrm{C}$-dots. Figure 4 shows the $\mathrm{UV}-\mathrm{Vis}$ absorption and emission spectra of the C-dots recovered by dissolution of sample 2 .

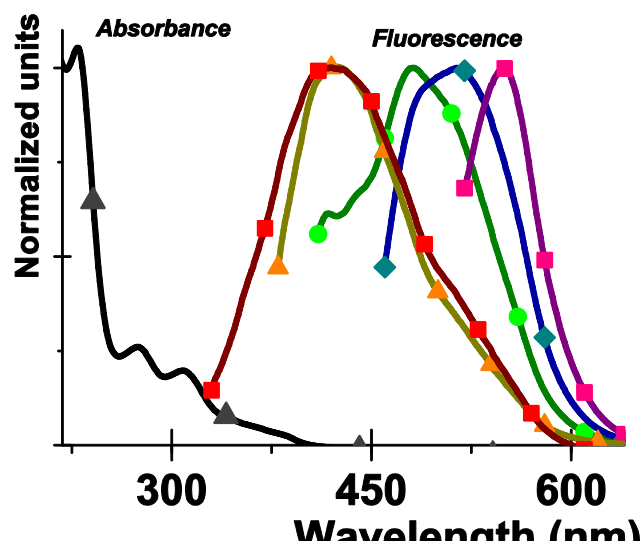

Figure 4. Absorption spectrum $(\boldsymbol{\Delta})$ and a set of emission spectra recorded upon excitation at $300(\boldsymbol{\Delta}), 350(\Delta)$, $400(\bullet), 450(\diamond)$ and $500(\square) \mathrm{nm}$ for the C-dots recovered by dissolution of sample 2 . The concentration of C-dots was about $0.1{\mathrm{mg} \times \mathrm{ml}^{-1}}^{-1}$ and baseline calibration was made using pure acetonitrile.

Figure 4 shows the absorption spectra of this organic extract that is characterized by the presence of four absorption bands, a broad band from 410 to $320 \mathrm{~nm}$ and three relative maxima at 308, 275 and $230 \mathrm{~nm}$, respectively. We noted that precedents in the literature do not show generally so well defined absorption bands for C-dots. ${ }^{8}$ It could be possible that formation of the C-dots by the pyrolysis in a confined space of the large 1.4 $\mathrm{nm}$ cavities could be responsible for the formation of C-dots with more uniform size compared to other preparation procedures in solution. There are precedents in the literature showing that homogeneous particle size in $\mathrm{MoS}_{2}$ of a few nm leads to defined absorption bands in contrast to the continuous absorption of mixtures of quantum dots of a broad size distribution. ${ }^{40,41}$

As expected, the material extracted with ethyl acetate was strongly photoluminescent and the position of emission maximum $\left(\lambda_{\mathrm{em}}\right)$ increases along the excitation wavelength in the range from 350 to $500 \mathrm{~nm}$ (Figure 4). Excitation wavelength also 
determines the Stokes shift and the broadness of the emission. The Stokes shift varies from 50 to $90 \mathrm{~nm}$ when the excitation wavelength is 350 and $400 \mathrm{~nm}$, respectively. In addition, the full width at half peak height ranges between $100 \mathrm{~nm}$ to $40 \mathrm{~nm}$ when the excitation wavelength is 400 and $500 \mathrm{~nm}$, respectively. As commented before this influence of the excitation wavelength on the photoluminescence spectrum is one of the main characteristics of the C-dots. ${ }^{8}$

Conclusive evidence in support of formation of C-dots by pyrolysis of the template trapped in ITQ-29 was obtained by high resolution transmission electron microscopy (HR-TEM) of the material after dissolution of the zeolite framework and extraction with ethyl acetate. Figure 5 shows selected HR-TEM images at increasing magnification of the organic content of sample 2. As it can be seen there, the sample is constituted by particles of size ranging from 5-9 $\mathrm{nm}$. This diameter is much larger than the one expected based on the dimension on the cavities of ITQ-29 (1.4 nm) and could reflect an increase in size during dissolution of the zeolite framework by $\mathrm{HF}$ and the extraction procedure. Alternatively, the dimensions of C-dots larger than the zeolite ITQ-29 cavities could be due to diffusion of the template inside the pores connecting several cavities as the $\mathrm{C}$-dot is being formed. In any case the images obtained by HRTEM provide firm support of the formation of C-dots upon pyrolysis of organic templates inside ITQ-29. Apparent agglomeration of the C-dots in Figure 5 has to be caused, at least

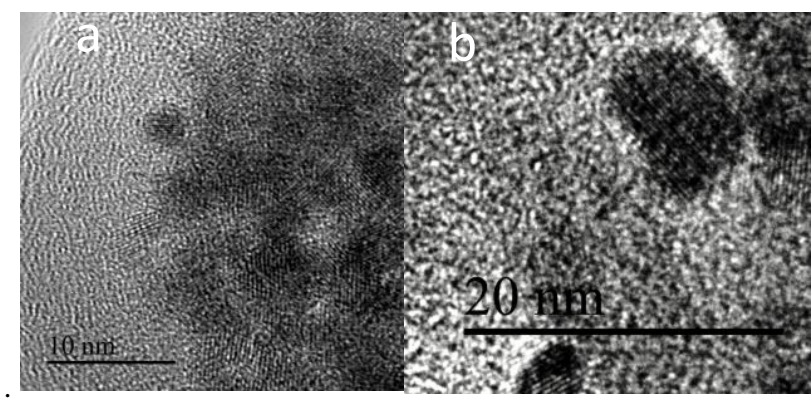

Figure 5. TEM images taken by drop casting on the carbon coated copper grid a sonicated acetonitrile suspension of C-dots from sample 2 submitted to pyrolysis at $550{ }^{\circ} \mathrm{C}$.

in part, by solvent evaporation when casting the sample onto the copper grid. In order to determine the real size of the particles in the suspension, dynamic laser scattering measurements of the $\mathrm{C}$ dots suspended in acetonitrile were carried out. A mononodal distribution centered at $380 \mathrm{~nm}$ was found. Since the dimension of particles in the liquid phase is much larger than the particle size determined by TEM, a degree of aggregation of C-dots in the suspension has to occur.

One of the open key issues in C-dots is the increase in the quantum yield of the photoluminescence. Typical values for
C-dot photoluminescence $\left(\Phi_{\mathrm{Pl}}\right)$ below 0.1 and in some cases emission quantum yield around 0.3 have been reported. ${ }^{8}$ In the present case, we have determined $\Phi_{\mathrm{Pl}}$ exciting at $350 \mathrm{~nm}$ and compared the emission intensity recorded for the C-dot with that of an optically matched solution of anthracene used as standard $\left(\Phi_{\mathrm{PL}}=0.3\right)^{42}$ the value obtained under this condition was $\Phi_{\mathrm{PL}}=0.4$ for the C-dots recovered after dissolving the zeolite. This $\Phi_{\mathrm{PL}}$ is relatively high for bare, raw $\mathrm{C}$-dots and $\mathrm{C}$-dot surface functionalisation by covalent attachment of polyethylenglycol units to passivate the surface has been reported to achieve quantum yields of $0.6 .^{8,43}$ It could be that $\Phi_{\mathrm{PL}}$ of the C-dots when encapsulated inside the rigid zeolite framework is even higher than 0.4 , although the lack of transparency of the solid powders makes not possible to quantitatively determine $\Phi_{\mathrm{PL}}$ before dissolution of the zeolite and extraction of C-dots- It should also be commented that according to chemical analysis, the C-dots obtained by pyrolysis of the quinolinium ion contains $\mathrm{N}$ atoms and that there is one recent precedent reporting that doping with $\mathrm{N}$ atoms increases $\Phi_{\mathrm{Pl} \cdot}{ }^{36}$ It is very likely that also here the high $\Phi_{\mathrm{Pl}}$ could be due to doping of the C-dots.

To address this issue, XPS analysis of the assynthesised ITQ-29 zeolite and the resulting sample 2 after 550 ${ }^{\circ} \mathrm{C}$ pyrolysis was performed. Figure 6 presents the set of experimental $\mathrm{C} 1 \mathrm{~s}$ and $\mathrm{N} 1 \mathrm{~s}$ peaks with the corresponding deconvolution to individual components, indicating their binding energies and their atomic proportion. Assignment of the components was based on the literature, ${ }^{4,}, 45$ although we notice that the binding energy for $\mathrm{C}$ atoms bonded to oxygen should be similar to the values for $\mathrm{C}$ atoms bonded to $\mathrm{N}$ that is the element present in the structure of the organic template (see Table 1). As expected, pyrolysis results in drastic changes in the XPS C1s and $\mathrm{N} 1 \mathrm{~s}$ peaks. Although the relative atomic composition of $\mathrm{C}$ (about $60 \%$ ) and $\mathrm{N}$ (about $2 \%$ ) does not change upon the thermal treatment, the nature of the $\mathrm{C}$ and $\mathrm{N}$ atoms undergoes a considerable rearrangement during the pyrolysis process. The component attributable to the positively charged ammonium $\mathrm{N}$ atoms present in sample $\mathbf{0}$ completely disappeared in the process and is absent in sample $\mathbf{2}$, in which some increase of the graphitic $\left(\mathrm{sp}^{2} \mathrm{~N}\right.$ atoms bonded to $\left.\mathrm{C}\right)$ and pyrrolic-type $\mathrm{N}$ atoms is observed. Also in the $\mathrm{C} 1 \mathrm{~s}$ peak the relative abundance of graphitic $\left(\mathrm{sp}^{2} \mathrm{C}\right.$ bonded to $\mathrm{C}$ ) measured for sample $\mathbf{0}$ decreases and is replaced by $\mathrm{C}$ atoms bonded to $\mathrm{O}$, or most probably, to $\mathrm{N}$ atoms. XPS data is relevant to show $\mathrm{N}$-doping of $\mathrm{C}$-dots and that a large proportion of $\mathrm{C}$ atoms should be bonded to $\mathrm{N}$ and $\mathrm{O}$ atoms. Thus, the origin of the photoluminescence could be charge separation $\mathrm{N}$ atoms acting as electron donors to electron vacancies due to the defects in the structure of the C-dot that is the commonly accepted mechanism in this type of nanomaterials. ${ }^{8}$ 

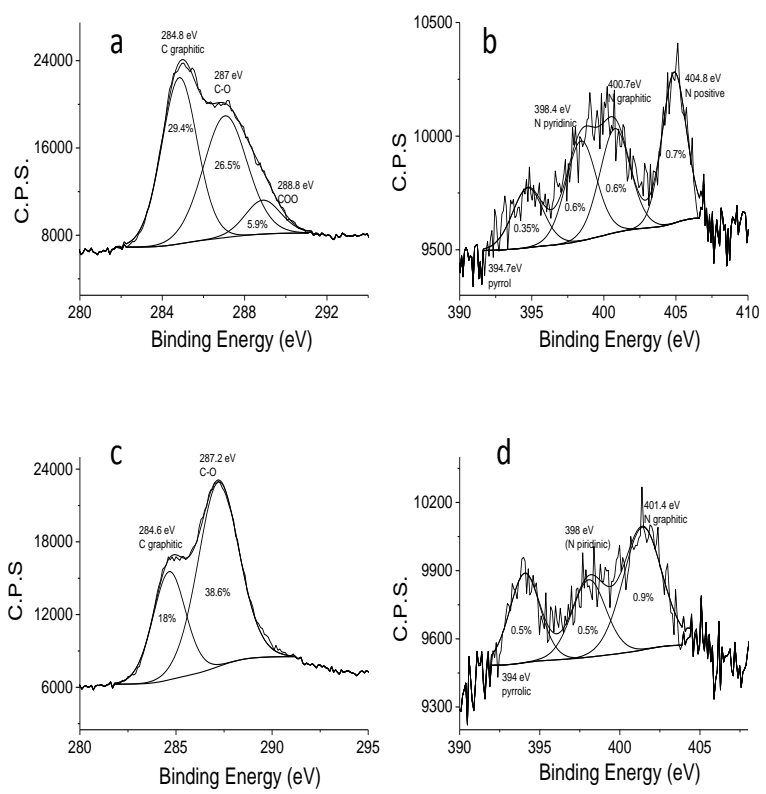

Figure 6. C1s (a and c) and N1s (b and d) XPS peaks and the best deconvolution into individual components measured for samples $\mathbf{0}$ ( $\mathrm{a}$ nd $\mathrm{b}$ ) and $\mathbf{2}$ (c and d).

The intense emission the C-dots resulting from pyrolysis of the zeolite ITQ-29 can be applied for the development of fluorescence sensors. Considering that the most likely photoluminescence mechanism is due to the $\mathrm{e}^{-} / \mathrm{h}^{+}$ recombination, oxygen should act as emission quencher due to the trapping of electrons that makes impossible charge recombination. Accordingly highly fluorescence $\mathrm{C}$-dots as the present case should serve as oxygen sensors that can find different applications for instance in food packaging and detection of leakage in sealed containers. ${ }^{46,}{ }^{47}$ In the present case we submitted an acetonitrile solution of C-dots derived from sample 2 to consecutive cycles of nitrogen and oxygen purging.

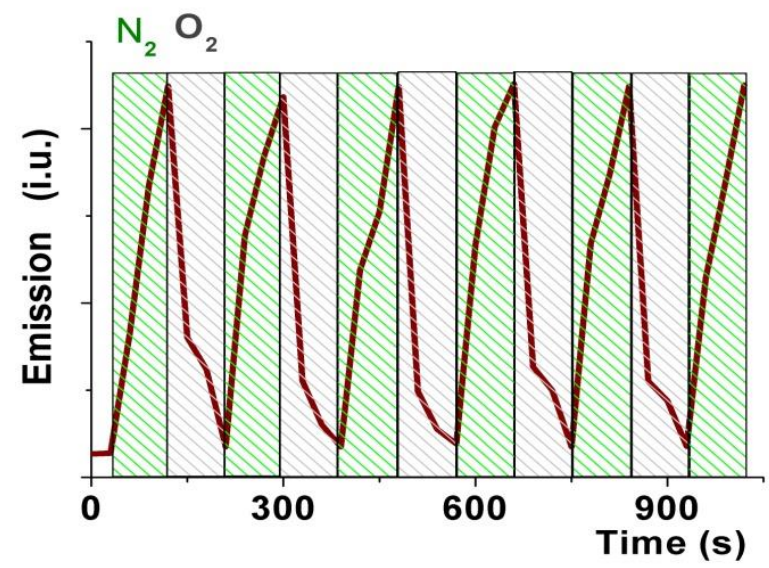

Figure 7. Variation of the emission intensity monitored at $500 \mathrm{~nm}\left(\lambda_{\mathrm{ex}} 350 \mathrm{~nm}\right)$ as a function of the nature $\left(\mathrm{N}_{2}\right.$ or $\left.\mathrm{O}_{2}\right)$ of the gas flow $\left(0.5 \mathrm{ml} \times \mathrm{min}^{-1}\right.$ of pure gas, either $\mathrm{N}_{2}$ or $\left.\mathrm{O}_{2}\right)$ measured for a quartz cuvette containing an acetonitrile solution of C-dots (optical density 0.3 at $350 \mathrm{~nm}$ ) recovered from zeolite ITQ-29 submitted to pyrolysis at $550{ }^{\circ} \mathrm{C}$.

Figure 7 shows the variation of the emission intensity monitored at $500 \mathrm{~nm}$ upon excitation at $350 \mathrm{~nm}$ depending on the nature of the gas flow $\left(1 \mathrm{ml} \mathrm{min}{ }^{-1}\right)$. As it can be seen there, $\mathrm{N}_{2}$ increases the intensity of the emission up to a value of $16.500,000 \pm 60,000$ counts, while the presence of $\mathrm{O}_{2}$ decreases the intensity to $12.340,000 \pm 20,000$ counts. This process of emission intensity increase and decrease is almost reversible and reproducible for consecutive cycles.

\section{Conclusions.}

In the present work we have shown that depending on the geometry and dimensions of the micropores as well the nature of the structure directing agent, small pore zeolites having large cavities and containing rigid quaternary ammonium ions can be submitted to pyrolysis at adequate temperatures to form highly photoluminescent $\mathrm{C}$-dots. In the present case, all-silica zeolite ITQ-29 has been found to be a suitable material for this purpose in contrast to medium and large pore size zeolites. After pyrolysis at $550{ }^{\circ} \mathrm{C}$ the resulting sample of zeolite ITQ-29 emits yellow photoluminescence upon illumination with blue light and even red photoluminescence upon illumination with green light. Entrapped C-dots can be recovered by $\mathrm{HF}$ dissolution of the zeolite frameworks and extraction with organic solvents. The photoluminescence quantum yield measured for the recovered $\mathrm{C}$ dots obtained at $550{ }^{\circ} \mathrm{C}$ pyrolysis was 0.4 and the distribution of the particle size ranged from 5 to $9 \mathrm{~nm}$. These C-dots can be used as fluorescent oxygen sensors. Considering the large number of zeolites known and the possibility to synthesise them in a wide range in the composition, the present results open the way for the search of the optimal zeolite for the preparation of highly emissive C-dots.

\section{Acknowledgements.}

Financial support by the Spanish Ministry of Economy and Competitiveness (Severo Ochoa and CTQ2012-32315) and Generalidad Valenciana (Prometeo 2012-014) is gratefully acknowledged.

\section{Experimental section.}

Synthesis of pure-silica zeolite ITQ-29. All the chemicals used have been provided by Aldrich. Pure silica zeolites ITQ29 , Beta ${ }^{48}$ and silicalite ${ }^{49}$ where prepared as reported. In particular, pure silica ITQ-29 zeolite (LTA structure) was synthesized in fluoride media according to a reported procedure in ref. ${ }^{39}$ from a gel of the following molar composition:

$\mathrm{SiO}_{2}: 0.25 \mathrm{ROH}: 0.25 \mathrm{TMAOH}: 0.5 \mathrm{HF}: 3 \mathrm{H}_{2} \mathrm{O}$

The gel were prepared by hydrolysing tetraethyl orthosilicate (TEOS) in an aqueous solution of 4-methyl-2,3,6,7-tetrahydro- 
1H,5H-pyrido[3.2.1-ij]quinolinium hydroxide (ROH) and tetramethylammonium hydroxide (TMAOH). The mixture was kept under stirring until the ethanol formed upon hydrolysis of TEOS and the appropriate excess of water was evaporated to reach the gel composition given above. Finally, an aqueous solution of HF (50\%) was added and the mixture was introduced in a Teflon-lined stainless autoclave and heated at $125{ }^{\circ} \mathrm{C}$ for 5 days. Then, the autoclave was cooled down, and the mixture was filtered, washed with distilled water and dried at $100{ }^{\circ} \mathrm{C}$ to yield the purely siliceous ITQ-29 zeolite.

Synthesis of ITQ-12. Pure silica ITQ-12 zeolite (ITW structure) was synthesized in fluoride media using 1,3,4trimethylimidazolium as organic structure directing agent, according to a reported procedure. ${ }^{50}$ The zeolite was prepared from a gel of the following molar composition: $\mathrm{SiO}_{2}: 0.56 \mathrm{ROH}$ : $0.56 \mathrm{HF}: 7 \mathrm{H}_{2} \mathrm{O}$. The gel was prepared by hydrolysing tetraethylorthosilicate (TEOS) in an aqueous solution of 1,3,4trimethylimidazolium hydroxide $(\mathrm{ROH})$. The mixture was kept under stirring until the ethanol formed upon hydrolysis of TEOS and the appropriate excess of water was evaporated to reach the gel composition given above. Finally, an aqueous solution of HF $(50 \%)$ was added and the mixture was introduced in a Teflonlined stainless autoclave and heated at $448 \mathrm{~K}$ for 7 days. Then, the autoclave was cooled down, and the mixture was filtered, washed with distilled water and dried at $100{ }^{\circ} \mathrm{C}$ to yield the purely siliceous ITQ-12 zeolite.

Formation of C-dots. The zeolite sample was compressed to 3 Ton $\mathrm{cm}^{-2}$ and the wafer was crushed and sieved, collecting the powder from 0.2 to $0.4 \mu \mathrm{m}$. Pyrolyses of these pelletized zeolite $(0.5 \mathrm{~g})$ were carried out in a vertical, tubular, quartz oven $(1.5 \mathrm{~cm}$ internal diameter) that was flushed with a nitrogen flow $(3 \mathrm{ml} / \mathrm{min})$. The temperature inside the reactor was measured with a thermocouple. Previously the zeolite samples were compressed to 1 Ton $\times \mathrm{cm}^{-2}$ for $2 \mathrm{~min}$ and the resulting wafer crushed and sieved. The zeolite powder was placed on a porous fitted glass inside the reactor and heated with an electrical furnace at $10{ }^{\circ} \mathrm{C} \times \mathrm{min}^{-1}$ rate from room temperature up to the required temperature that was maintained for $24 \mathrm{~h}$. At the end of pyrolysis, the sample was recovered and stored in ambient condition inside capped vials.

Recovery of C-dots by zeolite dissolution. Attempts to extract C-dots directly from the zeolite ITQ-29 with ethyl acetate were unsuccessful and it was necessary to dissolve the zeolite first. The zeolite $(50 \mathrm{mg})$ was dissolved at room temperature under magnetic stirring in $5 \mathrm{ml}$ aqueous $\mathrm{HF}$ solution (30 wt.\%) that was acidified with two drops of concentrated $\mathrm{HNO}_{3}$. After $24 \mathrm{~h}$, the solution was neutralised with $1 \mathrm{M} \mathrm{NaOH}$ solution and the resulting liquor extracted in a funnel with two aliquots of $5 \mathrm{ml}$ of ethyl acetate.

C-dots as oxygen sensor. An acetonitrile solution of C-dots having an optical density at $350 \mathrm{~nm}$ of 0.3 (about 0.1 $\mathrm{mg} \times \mathrm{ml}^{-1}$ ) in $1 \times 1 \mathrm{~cm}^{2}$ quartz cuvette with four polished sides capped with a septum was initially purged with pure oxygen 0.5 $\mathrm{ml} \times \mathrm{min}^{-1}$ for $3 \mathrm{~min}$ through needles. After this time the cuvette was place in the fluorimeter, measuring the emission intensity at $500 \mathrm{~nm}$ upon excitation at $350 \mathrm{~nm}$. Cycles of pure $\mathrm{N}_{2}(80 \mathrm{~s}, 0.5$ $\left.\mathrm{ml} \times \mathrm{min}^{-1}\right)$ followed by pure $\mathrm{O}_{2}\left(80 \mathrm{~s}, 0.5 \mathrm{ml} \times \mathrm{min}^{-1}\right)$ were performed. A plot of the emission intensity vs. times shows the changes in the photoluminescence intensity depending on the nature of the gas. These measurements were reproduce in duplicate.
Instrumental methods. Elemental chemical analyses of zeolite ITQ-29 samples either fresh or after being submitted to pyrolysis (Table 2) were carried out by combustion analysis using a Eurovector EA 3000 CHNS analyser using as catalyst a mixture of $\mathrm{Cu}$ and $\mathrm{WO}_{3}$. Thermogravimetric analysis were measured using a Mettler Toledo TGA/SDTA 851 thermobalance under air flow in the range of temperatures from ambient to 1000 ${ }^{\circ} \mathrm{C}$ at a temperature ramp of $10{ }^{\circ} \mathrm{C} \times \mathrm{min}^{-1}$. UV-Visible absorption spectra were recorded with a Jasco V-650 spectrophotometer. The solvent used in all measurements was dried HPLC quality $\mathrm{MeCN}$ and the spectra were recorded using $10 \mathrm{~mm} \times 10 \mathrm{~mm}$ quartz cells with a capacity of $4 \mathrm{ml}$. Baseline correction was made using pure acetonitrile and setting as zero the absorbance in all the spectral wavelength range. Fluorescence data were measured using $10 \mathrm{~mm} \times 10 \mathrm{~mm}$ quartz cells of $4 \mathrm{ml}$ total volume using a JACSO FP-8500 spectrofluorimenter. The absorbance of the sample was set at an optical density of 0.3 . The solutions were bubbled for $10 \mathrm{~min}$ with argon before the measurements. Optical microscopy images were captured using a Leica DM 4000 microscope with an adapted digital camera that has a series of filters that cut the illumination light above $400 \mathrm{~nm}$ (blue filter) or $550 \mathrm{~nm}$ (green filter) allowing also recording fluorescence images of the samples. XPS of the samples were obtained in a SPECS spectrometer with a 150-MCD9 detector using $\mathrm{MgK} \alpha(\mathrm{h} v=1253.6 \mathrm{eV}) \mathrm{X}$-ray radiation at an energy of 50 W. HRTEM images were recorded with a JEM $2100 \mathrm{~F}$ JEOL $200 \mathrm{kV}$ electronic microscope.

\section{Autors Adress.}

${ }^{1}$ Instituto Universitario de Tecnologia Quimica CSIC-UPV and Departamento de Química, Univ. Politecnica Valencia, Av. De los Naranjos S/N $46022 \quad$ Valencia. E-mail: hgarcia@qim.upv.es

2 Center of Excellence in Advanced Materials Research, King Abdulaziz University, Jeddah, Saudia Arabia

\section{References.}

1. B. K. An, S. K. Kwon, S. D. Jung, S. Y. Park, , Enhanced Emission and its Switching in Fluorescent Organic Nanoparticles. Journal of the American Chemical Society 2002, 124, (48), 14410-14415.

2. Y. F. Chen, Z. Rosenzweig, Luminescent CdS Quantum Dots as Selective Ion Probes. Analytical Chemistry 2002, 74, (19), 5132-5138.

3. S. Link, M. A. El-Sayed, Shape and Size Dependence of Radiative, Non-Radiative and Photothermal Properties of Gold Nanocrystals. International Reviews in Physical Chemistry 2000, 19, (3), 409-453.

4. M. Liong, J. Lu, M. Kovochich, Xia, T. Xia, S. G. Ruehm, A. E. Nel, F. Tamanoi, J. I. Zink, Multifunctional Iinorganic Nanoparticles for Imaging, targeting, and Drug Delivery. Acs Nano 2008, 2, (5), 889-896. 
5. I. L. Medintz, H. T. Uyeda, E. R. Goldman, H. Mattoussi, Quantum Dot Bioconjugates for Imaging, Labelling and Sensing. Nature Materials 2005, 4, (6), 435-446.

6. U. Resch-Genger, M. Grabolle, S. Cavaliere-Jaricot, R. Nitschke, T. Nann, Quantum Dots versus Organic Dyes as Fluorescent Labels. Nature Methods 2008, 5, (9), 763-775.

7. K. E. Sapsford, L. Berti, I. L. Berti, Materials for Fluorescence Resonance Energy Transfer Analysis: Beyond Traditional Donor-Acceptor Combinations. Angewandte ChemieInternational Edition 2006, 45, (28), 4562-4588.

8. S. Zhu, Q. Meng, L.Wang, Zhang, J. Zhang, Song, Y. Song, H. Jin, Zhang, K.; Sun, H.; Wang, H.; Yang, B., Highly Photoluminescent Carbon Dots for Multicolor Patterning, Sensors, and Bioimaging. Angewandte Chemie-International Edition 2013, 52, (14), 3953-3957.

9. $\quad$ Cao, L.; Wang, X.; Meziani, M. J.; Lu, F.; Wang, H.; Luo, P. G.; Lin, Y.; Harruff, B. A.; Veca, L. M.; Murray, D.; Xie, S.-Y.; Sun, Y.-P., Carbon Dots for Multiphoton Bioimaging. Journal of the American Chemical Society 2007, 129, (37), 11318.

10. Esteves da Silva, J. C. G.; Goncalves, H. M. R., Analytical and Bioanalytical Applications of Carbon Dots. TracTrends in Analytical Chemistry 2011, 30, (8), 1327-1336.

11. Shen, J.; Zhu, Y.; Yang, X.; Li, C., Graphene Quantum Dots: Emergent Nanolights for Bioimaging, Sensors, Catalysis and Photovoltaic devices. Chemical Communications 2012, 48, (31), 3686-3699.

12. Sun, Y. P.; Zhou, B.; Lin, Y.; Wang, W.; Fernando, K. A. S.; Pathak, P.; Meziani, M. J.; Harruff, B. A.; Wang, X.; Wang, H. F.; Luo, P. J. G.; Yang, H.; Kose, M. E.; Chen, B. L.; Veca, L. M.; Xie, S. Y., Quantum-Sized Carbon Dots for Bright and Colorful Photoluminescence. Journal of the American Chemical Society 2006, 128, (24), 7756-7757.

13. Sun, Y.-P.; Wang, X.; Lu, F.; Cao, L.; Meziani, M. J.; Luo, P. G.; Gu, L.; Veca, L. M., Doped Carbon Nanoparticles as a New Platform for Highly Photoluminescent Dots. Journal of Physical Chemistry C 2008, 112, (47), 18295-18298.

14. Wang, F.; Chen, Y.-h.; Liu, C.-y.; Ma, D.-g., White Light-Emitting Devices based on Carbon Dots' Electroluminescence. Chemical Communications 2011, 47, (12), 3502-3504.

15. Yang, S.-T.; Wang, X.; Wang, H.; Lu, F.; Luo, P. G.; Cao, L.; Meziani, M. J.; Liu, J.-H.; Liu, Y.; Chen, M.; Huang, Y.; Sun, Y.-P., Carbon Dots as Nontoxic and High-Performance Fluorescence Imaging Agents. Journal of Physical Chemistry C 2009, 113, (42), 18110-18114.

16. Atienzar, P.; Primo, A.; Lavorato, C.; Molinari, R.; Garcia, H., Preparation of Graphene Quantum Dots from Pyrolyzed Alginate. Langmuir 2013, 29, (20), 6141-6146.

17. Wang, Y.; Dong, L.; Xiong, R.; Hu, A., Practical Access to Bandgap-like N-doped Carbon Dots with Dual Emission Unzipped from PAN@PMMA Core-Shell
Nanoparticles. Journal of Materials Chemistry C 2013, 1, (46), 7731-7735.

18. Yang, Y.; Wu, D.; Han, S.; Hu, P.; Liu, R., Bottom-up Fabrication of Photoluminescent Carbon Dots with Uniform Morphology via a Soft-Hard Template Approach. Chemical Communications 2013, 49, (43), 4920-4922.

19. Liu, Y.; Xiao, N.; Gong, N.; Wang, H.; Shi, X.; Gu, W.; Ye, L., One-Step Microwave-Assisted Polyol Synthesis of Green Luminescent Carbon Dots as Pptical Nanoprobes. Carbon 2014, 68, 258-264.

20. Rajendran, R.; Sohila, S.; Muralidharan, R.; Muthamizhchelvan, C.; Ponnusamy, S., One-Pot Microwave Synthesis of Fluorescent Carbogenic Nanoparticles from Triton X-100 for Cell Imaging. European Journal of Inorganic Chemistry 2014, 2014, (2), 392-396.

21. Zhang, Y.-Y.; Wu, M.; Wang, Y.-Q.; He, X.-W.; Li, W.-Y.; Feng, X.-Z., A new Hydrothermal Refluxing Route to Strong Fluorescent Carbon Dots and its Application as Fluorescent Imaging Agent. Talanta 2014, 117, 196-202.

22. Habiba, K.; Makarov, V. I.; Avalos, J.; Guinel, M. J. F.; Weiner, B. R.; Morell, G., Luminescent Graphene Quantum Dots Fabricated by Pulsed Laser Synthesis. Carbon 2013, 64, 341-350.

23. Su, Y.; Xie, M.; Lu, X.; Wei, H.; Geng, H.; Yang, Z.; Zhang, Y., Facile Synthesis and Photoelectric Properties of Carbon Dots with Upconversion Fluorescence using ArcSynthesized Carbon by-Products. Rsc Advances 2014, 4, (10), 4839-4842.

24. Cundy, C. S.; Cox, P. A., The Hydrothermal Synthesis of Zeolites: History and Development from the Earliest Days to the Present Time. Chemical Reviews 2003, 103, (3), 663-701.

25. Cundy, C. S.; Cox, P. A., The Hydrothermal Synthesis of Zeolites: Precursors, Intermediates and Reaction Mechanism. Microporous and Mesoporous Materials 2005, 82, (1-2), 1-78.

26. Davis, M. E.; Lobo, R. F., Zeolite and Molecular-Sieve Synthesis. Chemistry of Materials 1992, 4, (4), 756-768.

27. Wang, Y.; Li, Y.; Yan, Y.; Xu, J.; Guan, B.; Wang, Q.; Li, J.; Yu, J., Luminescent Carbon Dots in a new Magnesium Aluminophosphate Zeolite. Chemical Communications 2013, 49, (79), 9006-9008.

28. Wang, Z.; Shi, W.; Lortz, R.; Sheng, P., Superconductivity in 4-Angstrom Carbon Nanotubes-a Short Review. Nanoscale 2010, 4, (1), 21-41.

29. Wang, Z.; Shi, W.; Xie, H.; zhang, T.; Wang, N.; Tang, Z.; Zhang, X.; Lortz, R.; Sheng, P.; Sheikin, I.; Demuer, A., Superconducting Resistive Transition in Coupled Arrays of 4 Angstrom Carbon Nanotubes. Physical Review B 2011, 81, (17).

30. Lortz, R.; Zhang, Q.; Shi, W.; Ye, J. T.; Qiu, C.; Wang, Z.; He, H.; Sheng, P.; Qian, T.; Tang, Z.; Wang, N.; Zhang, X.; Wang, J.; Chan, C. T., Superconducting Characteristics of 4Angstrom Carbon Nanotube-Zeolite Composite (vol 106, pg 
7299, 2009). Proceedings of the National Academy of Sciences of the United States of America 2009, 106, (22), 9121-9121.

31. Wang, Z.; Shi, W.; Xie, H.; zhang, T.; Wang, N.; Tang, Z.; Zhang, X.; Lortz, R.; Sheng, P.; Sheikin, I.; Demuer, A., Superconducting Resistive Transition in Coupled Arrays of 4 Angstrom Carbon Nanotubes. Physical Review B 2010, 81, (17).

32. Zhai, J. P.; Li, I. L.; Ruan, S. C.; Tang, Z. K., Fabrication of Smallest Single-Walled Carbon Nanotubes in Molecular Sieves: A Comparison between SAPO-11 and AlPO411. Microporous and Mesoporous Materials 2009, 124, (1-3), 15-19.

33. Zhai, J. P.; Peng, W. W.; Li, I. L.; Ruan, S. C.; Tang, Z. K., Study of the Mechanism of Carbonization of Template in Silicon-Substituted Aluminophosphate Zeolite Crystals. Journal of Physical Chemistry C 2008, 112, (31), 11702-11706.

34. Zhai, J. P.; Li, Z. M.; Liu, H. J.; Li, I. L.; Sheng, P.; Hu, X. J.; Tang, Z. K., Catalytic Effect of Metal Cations on the Formation of Carbon Nanotubes inside the Channels of AlPO4-5 Crystal. Carbon 2006, 44, (7), 1151-1157.

35. Li, Z. M.; Zhai, J. P.; Liu, H. J.; Li, I. L.; Chan, C. T.; Sheng, P.; Tang, Z. K., Synthesis of 4 Angstrom Single-Walled Carbon Nanotubes in Catalytic Si-Substituted AlPO4-5 Molecular Sieves. Applied Physics Letters 2004, 85, (7), 12531255 .

36. Dong, Y.; Pang, H.; Yang, H. B.; Guo, C.; Shao, J.; Chi, Y.; Li, C. M.; Yu, T., Carbon-Based Dots Co-doped with Nitrogen and Sulfur for High Quantum Yield and ExcitationIndependent Emission. Angewandte Chemie-International Edition 2013, 52, (30), 7800-7804.

37. Alvaro, M.; Cardin, D. J.; Colquhoun, H. M.; Garcia, H.; Gilbert, A.; Lay, A. K.; Thorpe, J. H., Complete Filling of Zeolite Frameworks with Polyalkynes Formed in situ by Transition-Metal Ion Catalysts. Chemistry of Materials 2005, 17, (10), 2546-2551.

38. Cardin, D. J.; Constantine, S. P.; Gilbert, A.; Lay, A. K.; Alvaro, M.; Galletero, M. S.; Garcia, H.; Marquez, F., Polymerization of Alkynes in the Channels of Mesoporous Materials Containing Ni and Zn Cations: Almost omplete Filling of the Voids. Journal of the American Chemical Society 2001, 123, (13), 3141-3142.

39. Corma, A.; Rey, F.; Rius, J.; Sabater, M. J.; Valencia, S., Supramolecular Self-Assembled Molecules as Organic Directing Agent for Synthesis of Zeolites. Nature 2004, 431, (7006), 287-290.

40. Wilcoxon, J. P.; Newcomer, P. P.; Samara, G. A., Synthesis and Pptical Properties of MoS2 and Isomorphous Nanoclusters in the Quantum Confinement Regime. Journal of Applied Physics 1997, 81, (12), 7934-7944.

41. Jeong, S.; Yoo, D.; Jang, J.-t.; Kim, M.; Cheon, J., Well-Defined Colloidal 2-D Layered Transition-Metal Chalcogenide Nanocrystals via Generalized Synthetic Protocols. Journal of the American Chemical Society 2012, 134, (44), 18233-18236.
42. Lakowicz, J. R., Principles of Fluorescence Spectroscopy. Springer: New York, 2006.

43. Wang, X.; Cao, L.; Yang, S.-T.; Lu, F.; Meziani, M. J.; Tian, L.; Sun, K. W.; Bloodgood, M. A.; Sun, Y.-P., BandgapLike Strong Fluorescence in Functionalized Carbon Nanoparticles. Angewandte Chemie International Edition 2010, 49, (31), 5310-5314.

44. Primo, A.; Atienzar, P.; Sanchez, E.; Delgado, J. M.; García, H., From Biomass Wastes to Large-Area, High-quality, N-Doped Graphene: Catalyst-Free Carbonization of Chitosan Coatings on Arbitrary Substrates. Chem. Commun. 2012, 48, 9254-9256.

45. Primo, A.; Sánchez, E.; Delgado, J. M.; García, H., High-Yield Production of N-Doped Graphitic Platelets by Aqueous Exfoliation of Pyrolyzed Chitosan. Carbon 2014, 68, (0), 777-783.

46. Bunn, H. F.; Poyton, R. O., Oxygen Sensing and Molecular Adaptation to Hypoxia. Physiological Reviews 1996, 76, (3), 839-885.

47. Hrapovic, S.; Liu, Y. L.; Male, K. B.; Luong, J. H. T., Electrochemical Biosensing Platforms Using Platinum Nanoparticles and Carbon Nanotubes. Analytical Chemistry 2004, 76, (4), 1083-1088.

48. Camblor, M. A.; Corma, A.; Valencia, S., Spontaneous nucleation and growth of pure silica zeolite- $\beta$ free of connectivity defects. Chem. Commum. 1996, 2365-2366.

49. Guth, J.L.; Kessler, H.; Wey, R., in Stud. Surf. Sci. Catal., Vol. 28, Y. Murakami, A. Iijima, J. W. Ward (eds.), Kodansha-Elsevier, Tokyo, 1986, p. 121.

50. Barrett, P. A.; Boix, T.; Puche, M.; Olson, D. H.; Jordan, E.; Koller, H.; Camblor, M. A. ITQ-12: A New Microporous Silica Polymorph Potentially Useful for Light Hydrocarbon Separations, Chem. Commum. 2003, 2114-2115. 
Table of Contents Graphic

\section{Highly fluorescent C-dots obtained by pyrolysis of quaternary ammonium ions trapped in all-silica ITQ-29 zeolite.}

Herme G Baldovi, ${ }^{1}$ Susana Valencia, ${ }^{1}$ Mercedes Alvaro, ${ }^{1}$ Abdullah M. Asiri ${ }^{2}$ and Hermenegildo Garcia ${ }^{1,2, *}$

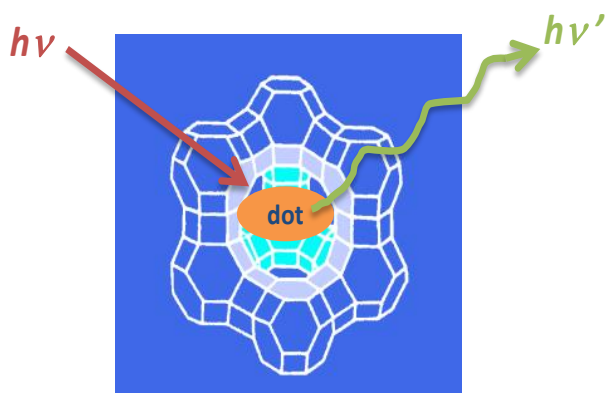

Pyrolysis of as-synthesised allsilica ITQ-29 zeolite leads to the formation of C-dots that exhibit an emission quantum yield of 0.4 